\title{
A retrospective analysis of the risk factors leading to perinatal mortality at a tertiary care hospital of Sikkim, India
}

\author{
Chanda Rai ${ }^{1 *}$, Latha V. Kharka ${ }^{1}$, Sudip Dutta², Nishant Kumar ${ }^{3}$
}

\begin{abstract}
${ }^{1}$ Department of Obstetrics and Gynecology, ${ }^{2}$ Department of Pediatrics, ${ }^{3}$ Department of Community Medicine, Sikkim Manipal Institute of Medical Sciences, Sikkim, India
\end{abstract}

\author{
Received: 22 March 2018 \\ Revised: 24 March 2018 \\ Accepted: 30 April 2018 \\ *Correspondence: \\ Dr. Chanda Rai, \\ E-mail: chanda17.rims@gmail.com
}

Copyright: () the author(s), publisher and licensee Medip Academy. This is an open-access article distributed under the terms of the Creative Commons Attribution Non-Commercial License, which permits unrestricted non-commercial use, distribution, and reproduction in any medium, provided the original work is properly cited.

\begin{abstract}
Background: This study helps to assess the burden of perinatal mortality at a tertiary referral hospital in Sikkim known for its hilly terrain which makes health services difficult to access. The aims and objectives were to determine the various causes and risk factors leading to perinatal mortality in order to formulate preventive strategies

Methods: All perinatal deaths over a year between August 2016-2017 were included and analysed in our study.

Results: A stillbirth rate of 14 per 1000 total births and early neonatal death rate of 8 per 1000 live births was found in 1855 total births. Complications related to pregnancy like pre-eclampsia (16\%), eclampsia (8\%), ante-partum haemorrhage $(15 \%)$ and medical disorders $(13 \%)$ were major contributors to stillbirths while pre-maturity $(53.3 \%)$, sepsis $(20 \%)$, birth asphyxia due to meconium aspiration (13.3\%) were notable factors leading to early neonatal deaths. In majority of the cases, factors like poor literacy, low socio-economic status, increased basal metabolic index and inadequate ante-natal care caused increased perinatal losses.

Conclusions: Perinatal grief continues to surround women who have suffered perinatal deaths and serious efforts should be made to bring down the mortality rates by improving health, nutrition of all expecting mothers and increase their awareness to seek ante-natal health services in order to avoid any catastrophe in terms of maternal and fetal loss.
\end{abstract}

Keywords: Early neonatal deaths, Maternal risk factors, Perinatal mortality, Stillbirths

\section{INTRODUCTION}

Perinatal mortality rate (PMR) reflects the health care system of a nation and emphasises the obstetric and pediatric care provided to all mothers and neonates. ${ }^{1}$ Perinatal mortality includes all stillbirths i.e. fetuses born with no signs of life after 28 weeks of gestation or weighing 1000 grams or more and neonatal deaths within the first seven days of life. ${ }^{2}$

Worldwide, there are 6.3 million perinatal deaths a year, more than half of them occurring in developing countries and $27 \%$ of them in least developed countries. 2 The perinatal mortality rate is five times higher in developing countries than in developed regions with 10 per 1000 total births in developed countries, 50 per 1000 in developing countries and over 60 per 1000 in least developed countries. PMR in Asia is 50 per 1000 births. $^{2}$ India contributes significantly to the global burden with a perinatal mortality rate of 23 per 1000 total births (SRS data 2016). India with 5.92 lac stillbirths account for $22.6 \%$ of the global burden of stillbirths in $2015 .^{3}$

WHO formulated the Every Newborn Action Plan (ENAP) to reduce stillbirth rate to less than 12 per 1000 births by $2030.4^{4}$ On these lines, India adopted Indian 
Newborn Action Plan (INAP) to achieve single digit stillbirth rate by the year $2030 .{ }^{3}$ In developed countries stillbirth rate has fallen with an annual reduction rate of $2 \%$ but the rate of decline of stillbirths in India is not at the same rate as that of maternal and neonatal mortality. ${ }^{4}$

This study is an effort to assess the magnitude of problems in our hospital in order to design preventive strategies.

\section{Aims of the study}

- To determine the various causes of stillbirths and early neonatal deaths.

- To identify the risk factors causing stillbirths and early neonatal deaths.

- To frame preventive strategies to reduce the perinatal mortality rate.

\section{METHODS}

It was a retrospective observational study done at Central Referral Hospital of Sikkim Manipal Institute of Medical Sciences which is a tertiary care hospital.

\section{Inclusion criteria}

All perinatal deaths between August 2016-17 were included in this study.

\section{Data collection}

The details of the women which included their sociodemographic profile, the ante-natal, ante-partum and intra-partum period and all risk factors leading to perinatal deaths were analysed.

\section{Outcome measure}

Stillbirth rate $(S B R)$

SBR was calculated as number of stillbirths per 1000 births.

\section{Early neonatal death rate (ENDR)}

ENDR measured as number of early neonatal deaths or deaths within first seven days of life per 1000 births.

\section{Perinatal mortality rate (PMR)}

PMR calculated as total number of stillbirths and early neonatal deaths per 1000 total births.

\section{RESULTS}

Our results showed that out of 1855 live births, there were 26 cases of stillbirths which gave a stillbirth rate of 14 per 1000 and 15 cases of early neonatal deaths with a rate of 8 per 1000 births contributing to total 41 cases of perinatal deaths with a PMR of 22 per 1000 births. Out of 26 cases of stillbirths there were 24 cases of macerated stillborn babies and only two cases of fresh stillborn babies (Figure 1).

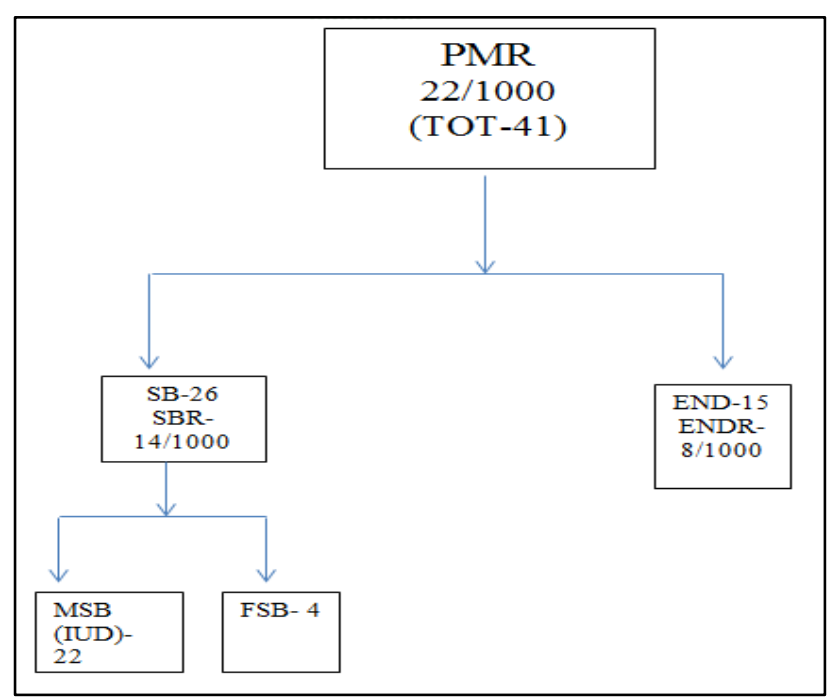

Figure 1: Rate of perinatal mortality.

Maximum contributors to stillbirths in our study are hypertensive disorders of pregnancy followed by antepartum haemorrhage and medical disorders (Figure 2).

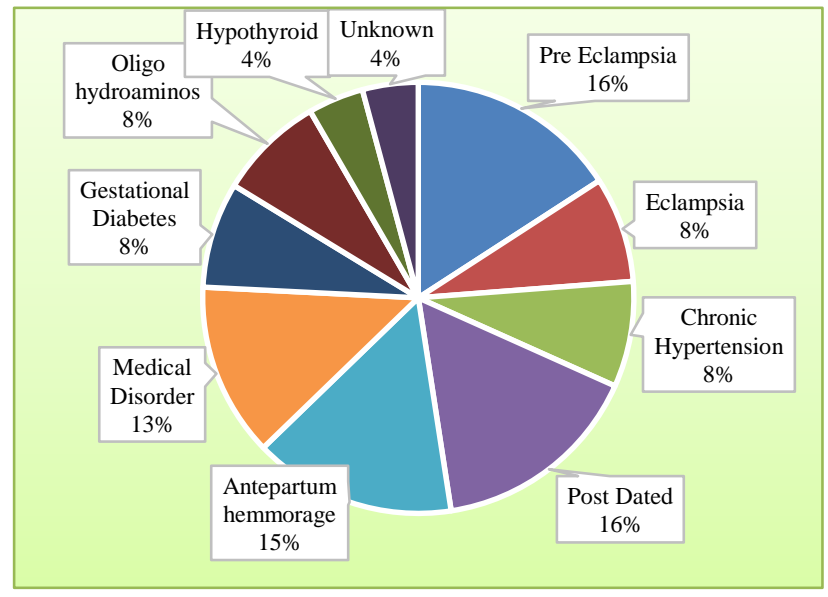

Figure 2: Causes of still birth.

More than $50 \%$ causes of early neonatal deaths were due to prematurity followed by sepsis, meconium aspiration, congenital anomalies and intra-uterine growth restriction (Figure 3).

We tried to analyse the risk factors (Table 1) leading to perinatal mortality and found that based on age, $57.5 \%$ of young women in the age group 18-25 years and $32.5 \%$ older women more than 35 years of age had more perinatal deaths. More perinatal losses occurred in multiparous women $(61 \%)$ as compared to primi-parous women $(39 \%)$. 


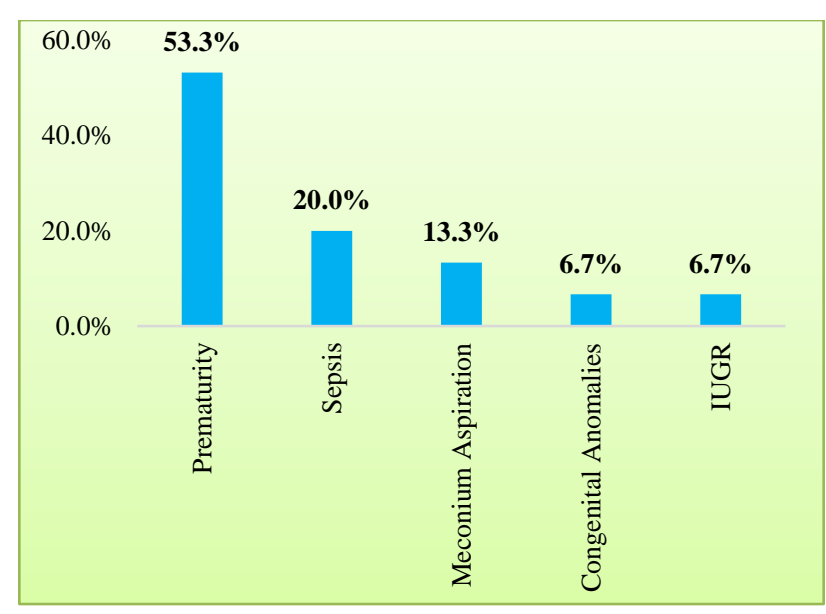

Figure 3: Causes of ENDs.

Table 1: Determinants of perinatal mortality.

\begin{tabular}{|ll|}
\hline Variable & $\mathrm{n}(\%)$ \\
\hline Age & $24(59.5)$ \\
\hline 18-25 years & $3(8.0)$ \\
\hline $26-34$ years & $14(32.5)$ \\
\hline 35 and above & \\
\hline Parity & $25(61)$ \\
\hline Primigravida & $16(39)$ \\
\hline Multigravida & \\
\hline Type of registration & $8(19.5)$ \\
\hline Booked & $30(73.2)$ \\
\hline Referred & $3(7.3)$ \\
\hline Un booked & $26(64)$ \\
\hline Socio economic status & $15(36)$ \\
\hline Low & \\
\hline High & $22(55)$ \\
\hline BMI & $19(44)$ \\
\hline $18-25 \mathrm{~kg} / \mathrm{m}^{2}$ & \\
\hline $25-$ above & $23(56)$ \\
\hline Literacy status & $18(44)$ \\
\hline Primary or less & \\
\hline Above primary & $28(69)$ \\
\hline Number of ANC Visits & $13(31)$ \\
\hline Less than 3 & $19(46.3)$ \\
\hline 3 or more & $3(7.3)$ \\
\hline POG & $19(46.3)$ \\
\hline$<34$ weeks & $28(68.3)$ \\
\hline $35-40$ weeks & $13(31.7)$ \\
\hline$>40$ weeks & \\
\hline Birth weight & \\
\hline$<2.5$ Kgs & \\
\hline$>2.5 \mathrm{kgs}$ & \\
\hline
\end{tabular}

Socio-economic status showed that women belonging to lower socio-economic status (83.4\%) had adverse outcomes. This was reflected in the basal metabolic index (BMI) with $55 \%$ of the patients who were undernourished with BMI $<18$ and $45 \%$ were in the obese category with $\mathrm{BMI}>30$.
Women who were illiterate or had less than primary education (74\%) had poor fetal outcomes. Patients with less than three ante-natal visits $69 \%$ ) had poor fetal outcomes. Since ours is a tertiary referral hospital, most of the patients coming to us were either referred $(73.2 \%)$ or unbooked $(19.5 \%)$ with a small percentage of our booked patients. One of these patients had uncontrolled diabetes and had term intra-uterine death and the other had intrapartum death due to meconium aspiration.

Perinatal deaths were seen more at gestational age <34 weeks and at $>40$ weeks indicating that prematurity and post-datism remain major contributors to PMR.

Babies born with birth weight less than $2.5 \mathrm{~kg}$ either due to prematurity or due to growth restriction had adverse outcomes $(68.3 \%)$ in our study.

\section{DISCUSSION}

PMR in our study is 22 per 1000 births which is slightly less as compared to national statistics of 23 per 1000 (SRS data 2016). This study reflects the fact that pregnancy associated conditions like pre-eclampsia, eclampsia, ante-partum haemorrhage and other medical disorders contribute majorly to all the stillbirths. Factors like pre-maturity, sepsis and birth asphyxia due to meconium aspiration are causing early neonatal losses. Women having perinatal deaths in our study were found to belong to younger age group, with poor socioeconomic status, were under-nourished or anemic. Most of them did not complete their secondary education, and did not avail regular ante-natal health check-ups due to poor accessibility to health centre or other reasons.

This is further emphasised in study done by Sharmila B et al who found that over the past fifty years in India, 3 major causes of neonatal deaths are complications of preterm births (35\%), infections (33\%) and birth asphyxia or intra-partum related conditions $(20 \%) .^{2}$ They also stated that prevalent maternal factors resulting in increased perinatal loss include adolescent pregnancy, maternal malnutrition, poor socio-economic status, anemia, lack of ante-natal care, maternal infection, preeclampsia and diabetes. ${ }^{2}$

A study was conducted by Rahman $\mathrm{H}$ et al to assess the determinants of stillbirths at our centre between 20022011 and they found that overall stillbirth rate was 26.49 per 1000 births over the decade. ${ }^{5}$ It was noted that obstetric complications like pre-eclampsia, eclampsia, ante-partum haemorrhage and preterm labour are major causes of perinatal losses and these are largely avoidable causes if there is increased awareness about routine antenatal follow-up, timely detection and referral so that necessary intervention can be done at an appropriate time to avoid any catastrophe in terms of fetal and maternal health. The stillbirth rate has come down drastically to 14 per 1000 births in our study which indicates the improvement in terms of good ante-natal care and 
efficient monitoring during intrapartum process of labour which has remarkably reduced over the years. It was observed in our study that maternal factors like age, literacy status and nutritional status greatly influence PMR. Teenage pregnancy rate is very high in Sikkim and most of the young women in our study had hypertensive disorders which resulted on perinatal deaths. Sankar MJ et al did a study on Indian newborns and found that age $<20$ is associated with increased PMR. ${ }^{6}$ It is estimated that neonatal mortality rate would reduce by $9.4 \%$ if age at childbirth is raised to above $20 .^{7,8}$

Women with age more than 35 in our study suffered from various medical disorders of pregnancy like thyroid disorder, essential hypertension, diabetes which amounted to greater perinatal loss.

Sikkim has a very high school dropout rate and most of the women in our study did not attain secondary education. This is reflected in their poor availment of health services. Women with low BMI had more incidence of anemia indicating poor nutritional status.

Located in the Himalayas, the state of Sikkim is characterised by its mountainous terrain. Accessibility to health care is a major deterrent in our state because most of the areas are remote from our centre and the hilly terrain makes it difficult to come for regular visits. These patients seek ante-natal care at local dispensary or primary health centres and when they are referred to us sometimes mishaps are unavoidable in spite of our best efforts.

Next, we need to stress on maternal education as educating her would increase her awareness towards health services available hence increasing their utilization. The triad of low socio-economic status, illiteracy and inadequate ante-natal care are major contributors to stillbirths. ${ }^{9-11}$

Best example is Kerala which has already achieved single digit PMR of 9/1000 births based on high literacy levels. Women with low BMI in our study had more incidence of anemia indicating poor nutritional status. It is essential to improve maternal nutritional status to reduce the incidence of low birth weight babies and preterm childbirth. The Integrated Child Development Scheme aims to provide free ration to all ante-natal women. We need to make the availability of health services more uniform in distribution and improve their infrastructure and provide more manpower.

Ground level workers like ASHA (Accredited Social Health Activist) need to be formally trained in order to identify high risk cases so as to refer them to a tertiary health facility at the earliest possible time. Initiatives like Janani Suraksha Yojana need to be closely monitored for better outputs.

\section{CONCLUSION}

The onus is on the national and the state governments, the society on a whole and the health institutions to formulate new policies to enable to achieve the target of achieving single digit PMR by 2030. INAP has introduced six intervention packages which lays added stress on antenatal care which needs strict adherence protocols. We need to work not only for the figures but also to alleviate the perinatal grief which remains a less discussed issue.

Funding: No funding sources

Conflict of interest: None declared

Ethical approval: The study was approved by the Institutional Ethics Committee

\section{REFERENCES}

1. PMR Reflects Health Status: WHO library Cataloguing in Publication. Data neonatal \& perinatal mortality: Country, regional and global estimates. 2006.

2. Mukherjee SB, Bandhopadhyay T. Perinatal mortalitywhat has changed: Indian Pediatr. 2016;153:242-3.

3. Roy MP. Mitigating the stillbirth challenge in India. The Lancet. 2016;387(10032): 1995.

4. Lawn JE, Blencowe H, Waiswe P. And for the Lancet ending preventable stillbirths series study group with the Lancet stillbirth epidemiology investigator group. Stillbirths: rates, risk factors, and acceleration towards 2030. Lancet. 2016;387:587-603.

5. Rahman H, Dutta S, Choudhury P, Kar S, Nandi P. (2014). Determinants of stillbirths in a teaching hospital of Sikkim Province of India. J Womens Health Issues Care. 2014;3:5

6. Sankar MJ, Neogi SB, Sharma J, Chauhan M, Srivastava R, Prabhakar PK, et al. State of newborn health in India. J Perinatol. 2016;36(Suppl 3):S3-8.

7. Paul VK, Sachdev HS, Mavalankar D, Ramachandran P, Sankar MJ, Bhandari N, et al . Reproductive health and child health and malnutrition in India: meeting the challenge. Lancet 2011;377:332-349.

8. Conde- Agudelo A, Belizan JM, Lammers C. Maternal -perinatal morbidity and mortality associated with adolescent pregnancy in Latin America: cross-sectional study. Am J Obstet Gynecol. 2005;192(2):342-9.

9. Korde- Nayak, VN and Gaikwad, PR. Causes of stillbirth. J Obstet Gynecol India. 2008;58:314-8.

10. Blencowe H, Cousens S, Jassir FB, Say L, Chou D, Mathers C, et al. National, regional, and worldwide estimates of stillbirth rates in 2015 , with trends from 2000: a systematic analysis. Lancet Glob Health. 2016;4:e98-108.

Cite this article as: Rai C, Kharka LV, Dutta S, Kumar N. A retrospective analysis of the risk factors leading to perinatal mortality at a tertiary care hospital of Sikkim, India. Int J Reprod Contracept Obstet Gynecol 2018;7:2295-8. 